\title{
Asbestos exposure and primary pleural lymphoma
}

\begin{abstract}
Pleural involvement in systemic lymphoma is common but primary pleural lymphomas are very rare. Current literature describes two types; the primary effusion lymphoma of patients with human immunodeficiency virus (HIV) and the pleural lymphoma of patients with tuberculosis. We report a case of primary pleural large B cell lymphoma in a 77 year old gentleman. He was a lifelong non-smoker with no history of tuberculosis or HIV infection, but significant exposure to asbestos. Our case highlights an increasing association in the literature between primary pleural lymphoma and asbestos exposure. It provides a novel insight into the pathogenesis of primary pleural lymphoma and provides an important differential diagnosis for asbestos related pleural mesothelioma.
\end{abstract}

Keywords: immunocytopathology, primary pleural lymphoma, thoracic oncology, epstein-barr virus

\author{
Volume I Issue 2 - 2015
}

\author{
Miguel A Perez-Machado, Alexandra Pain \\ Department of Cellular Pathology, Royal Free Hampstead NHS \\ Trust, London
}

\begin{abstract}
Correspondence: Alexandra Pain, Department of Cellular Pathology, Royal Free Hampstead NHS Trust, Pond Street London NW3 2QG, London, Tel 07540060297, Email alexandra.pain.10@ucl.ac.uk
\end{abstract} Received: August 27, 2015 | Published: September 18,
2015

\section{Introduction}

Although pleural involvement in systemic lymphoma is common, primary pleural lymphomas are very rare. Two types have been described; the primary effusion lymphoma of patients with human immunodeficiency virus (HIV) and the pleural lymphoma of patients with tuberculosis, secondary to either a chronic pyothorax or treatment by inducing an artificial pneumothorax. ${ }^{1,2}$ We report a case of large B cell lymphoma of the pleura, occurring in an immunocompetent patient with no history of tuberculosis. This case highlights an increasing association between primary pleural lymphoma and asbestos exposure.

\section{Case history}

A 77 year old male nursing home resident presented a four week history of dyspnoea, non-productive cough, progressive dysphagia and generalised fatigue. He denied any appetite change or weight loss, fevers or night sweats, chest pain, haemoptysis or wheeze. He was a lifelong non-smoker, exposed to asbestos whilst working in a shoe factory. Clinical examination revealed decreased breath sounds and crackles in the left lower zone, but no peripheral lymphadenopathy or hepatosplenomegaly. Computerised tomography of the chest, abdomen and pelvis was carried out (Figure 1). Bilateral pleural effusions were seen, with left lower lobe consolidation. Meditational and right hilar lymphadenopathy compressing the oesophagus was noted and pleural calcifications suggested previous asbestos exposure.

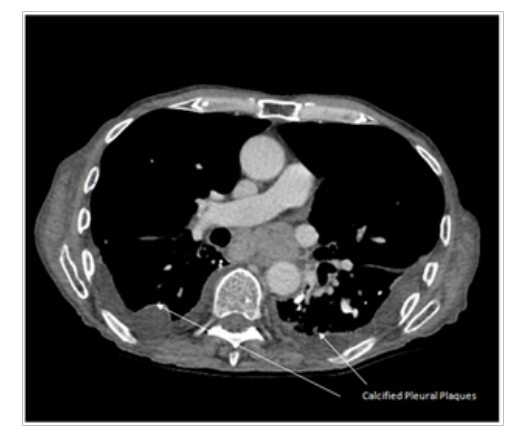

Figure I Computerised tomography of the chest showing bilateral pleural effusions and meditational lymphadenopathy.
Cytological examination of the pleural effusion showed a malignant lymphomatous effusion. Numerous lymphoid cells were seen, with scant delicate cytoplasm and high nuclear: cytoplasmic ratio (Figure 2A). Nuclear abnormalities included irregular nuclear membranes (cleave and nuclear knobs) and coarse chromatin with prominent nucleoli. Mitotic activity was brisk with occasional abnormal mitotic figures. A lack of fibrin exudate and mixed inflammatory cells, together with the increased mitotic activity and nuclear abnormalities favoured a diagnosis of malignant lymphomatous effusion. A diffuse large B cell lymphoma was suspected and investigations were expanded. Immunophenotyping showed the cells to be positive for CD20, CD45, CD138, bcl-2, bcl-6, MUM-1 and MIB-1 (>90\%) and negative for HHV8 (human herpes virus 8), EBV (Epstein-Barr virus), CD3, CD5 and CD10: this was confirmed by flow Cytometry (Figures 2B-2E). These findings were in keeping with the diagnosis of diffuse large $\mathrm{B}$ cell lymphoma.

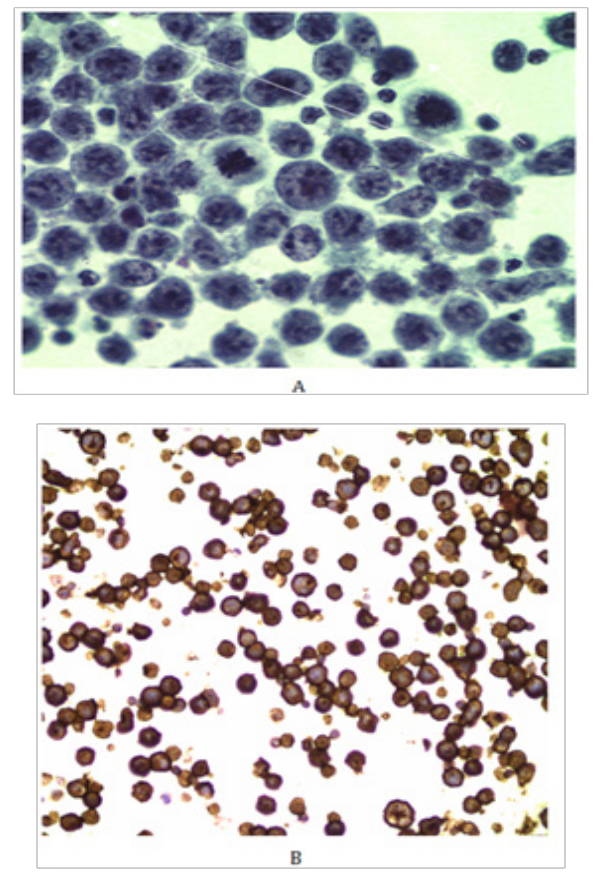



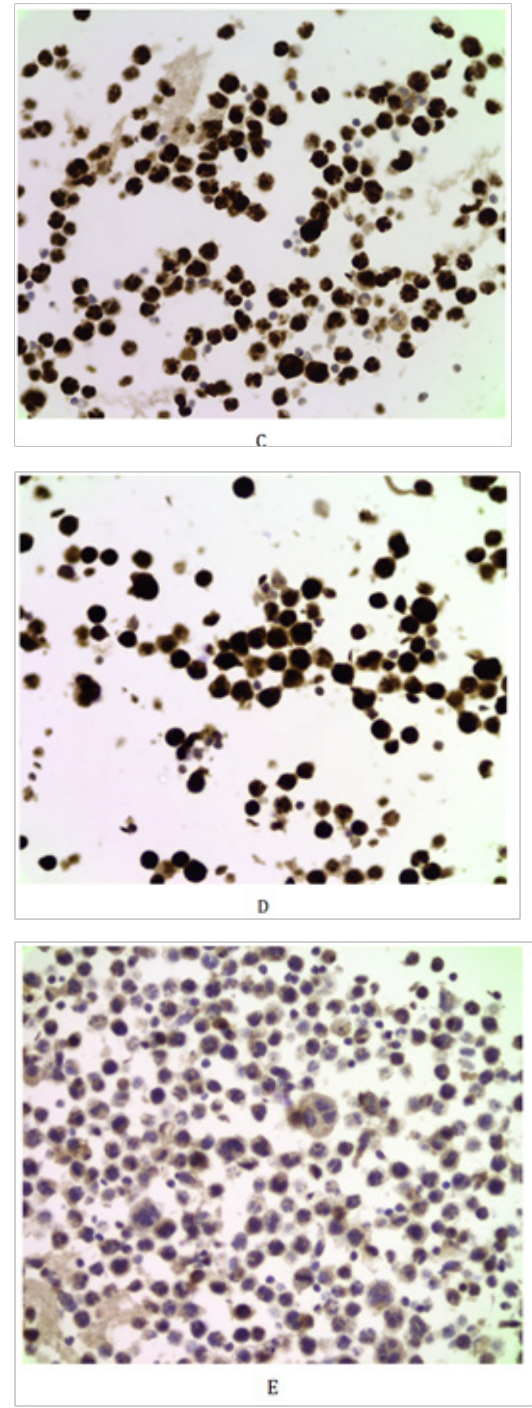

Figure 2 Cytology evaluation of pleural fluid showing A) numerous lymphoid cells with scant cytoplasm, irregular nuclear membranes and coarse chromatin, plus several mitotic bodies (Papanicolaoux00). Immunohistochemistry showing the majority of cells positive for B) CD20 (x40), C) MIB-I (x40), D) MUM-I (x40), E) CDI38 (x40).

Pleural biopsy showed cores of fibro connective tissue and patchy infiltrate of atypical lymphoid cells, with irregular hyperchromatic nuclei. Immunohistochemical staining showed CD20, bcl-2, bcl-6, MUM-1 and MIB-1 (50\%) positive cells, negative for CD10. This was consistent with a diagnosis of primary diffuse large B cell lymphoma of the pleura, non-germinal centre type. A bone marrow aspirate and trephine showed normal cellularity, but a large focus of cells positive for CD20 and PAX-5 were seen, suggesting focal involvement by diffuse large B cell lymphoma. The patient was treated with chemotherapy. Sadly, after several admissions for neutropenic sepsis, the decision was made that no further chemotherapy would be given and the patient died one year after diagnosis.

\section{Discussion}

Pleural involvement in systemic lymphoma is common; approximately $16 \%$ of patients with non-Hodgkin's lymphoma develop pleural involvement during the course of their disease. ${ }^{3}$ However, primary pleural lymphoma is extremely rare, making up $2.4 \%$ of primary chest wall tumours. ${ }^{4}$ The current literature delineates two types, both clinically aggressive and well described pathologically and clinically. The first is a primary effusion lymphoma, characterised by a lymphomatous effusion in the absence of a mass, often occurring in patients with HIV. ${ }^{1} \mathrm{HHV} 8$ is always implicated and EBV infection is present in $50 \%$ of patients. Patients with a history of tuberculosis may develop pleural lymphoma, presenting with a mass lesion, secondary to either a chronic tubercular pyothorax or treatment by inducing an artificial pneumothorax. ${ }^{2}$ It is hypothesised that chronic inflammation leads to chronic stimulation of B lymphocytes in the pleural cavity: the most common pleural lymphoma is reported to be diffuse large B cell non-Hodgkin's lymphoma, supporting this hypothesis. ${ }^{5} \mathrm{EBV}$ infection is present in $100 \%$ of these patients. Primary pleural lymphoma in an immunocompetent patient, with no history of tuberculosis, is exceedingly rare and to our knowledge, few previous cases have been reported. A theme in the current literature was noted: in patients with primary pleural lymphomas, non-tuberculosis or HIV related, there was often a history of previous asbestos exposure. ${ }^{6-8}$ The link between asbestos and mesothelioma is well known and an association between asbestos exposure and lymphoid malignancy has also been noted in the literature. ${ }^{9}$ This case adds to the growing evidence that asbestos could be associated with primary pleural lymphoma. It has been postulated that by causing intense chronic stimulation of B lymphocytes and a decrease in numbers of $\mathrm{T}$ lymphocytes, asbestos exposure leads to lymphoid, especially B lymphocyte, hyperplasia, a well-known precursor to malignancy. This provides a novel insight into the pathogenesis of primary pleural lymphoma, as well as providing an important differential diagnosis for asbestos related pleural mesothelioma.

\section{Acknowledgements}

None.

\section{Conflict of interest}

The author declares no conflict of interest.

\section{References}

1. Mitchell A, Meunier C, Ouellette D, et al. Extranodal marginal zone lymphoma of mucosa-associated lymphoid tissue with initial presentation in the pleura. Chest. 2006;129(3):791-794.

2. Nakatsuka S, Yao M, Hoshida Y, et al. Pyothorax-associated lymphoma: a review of 106 cases. J Clin Oncol. 2002;20(20):4255-4260.

3. Vega F, Padula A, Valbuena JR, et al. Lymphomas involving the pleura Arch Pathol Lab Med. 2006;130(10):1497-1502.

4. King RM, Pairolero PC, Trastek VF, et al. Primary chest wall tumors factors affecting survival. Ann Thorac Surg. 1986;41(6):597-601.

5. Hirai S, Hamanaka Y, Mitsui N, Morifuji K, et al. Primary malignant lymphoma arising in the pleura without preceding long-standing pyothorax. Ann Thorac Cardiovasc Surg. 2004;10(5):297-300.

6. Sabit R, Bolton CE, Attanoos R, et al. Non-Hodgkin's lymphoma of the pleural cavity masquerading as pleural mesothelioma. Respiratory Medicine Extra. 2005;1(4):144-147.

7. Ahmad H, Pawade J, Falk S, et al. Primary pleural lymphomas. Thorax 2003;58(10):908-909. 
8. Parisio E, Bianchi $\mathrm{C}$, Rovej R, et al. pulmonary asbestosis associated to pleural non-Hodgkin lymphoma. Tumori. 1999;85(1):75-77.
9. Jacobson RJ, Memoli D, Kagan E. Malignant lymphomas in asbestos workers: clinico-pathological features and concerns about bleomycin. Hematol Rev Commun. 1990;4:217-27. 